\title{
Research on Time Synchronization Method in WSN
}

\author{
Lusha Zhang ${ }^{1}$, Jialin $\mathrm{Zhu}^{2, *}$ and Chunli $\mathrm{Gao}^{3}$ \\ ${ }^{1}$ School of Automation, Beijing Information Science and Technology University, Beijing 100020, China \\ ${ }^{2}$ Electronic Information and Control Experimental Teaching Center, Beijing Information Science and Technology University, \\ Beijing 100020, China \\ ${ }^{3}$ School of Automation, Beijing Information Science and Technology University, Beijing 100020, China \\ ${ }^{*}$ Corresponding author
}

\begin{abstract}
WSN is a typical distributed system. Data fusion, energy management, transmission scheduling, target tracking and positioning, and network security all require monitoring nodes in the wireless sensor network to maintain time synchronization. The application of synchronization technology at this stage is discussed. The clock model of the nodes in WSN is given, and the basic types of time synchronization technology are introduced respectively: RR mechanism, SR mechanism, RO mechanism, and hybrid synchronization mechanism. The principle of synchronization mechanism of different time synchronization algorithms in each mode is analyzed. The application scope, advantages and disadvantages are analyzed. The error analysis is simulated, the requirement of wireless sensor network for time synchronization is analyzed, and the existing time synchronization technology is compared. Finally, the HRTS algorithm is selected as the synchronization mode of the WSN network.
\end{abstract}

Keywords-WSN; time synchronization; error analysis; HRTS

\section{INTRODUCTION}

At present, some mature time synchronization protocols have been formed at home and abroad [1]. The traditional network time synchronization technology is NTP (Network Time Protocol, Network Time Protocol) and SNS (Satellite Navigation System). It has been widely used in wired networks such as the Internet, and its latest synchronization accuracy reaches microseconds. However, its complex operation, high hardware environment requirements, limited accuracy, and relatively stable network architecture are not suitable for handling dynamic wireless sensor networks. GPS global positioning system combined with wireless ranging technology can achieve the nanosecond accuracy of the entire network, but its high cost and energy consumption can not be used in high pressure, high magnetic and complex environments.

WSN is an abbreviation of Wireless Sensor Network, which is a wireless sensor network. Its components are sensors, sensing objects, and clients[2]. The WSN wireless sensor network collects signals through the sensor nodes at the front end, and then sends the detected information to the users by wireless communication. In its various applications, the system requires high precision for timing and must ensure the timing synchronization of each monitoring node. Therefore, it is critical to study time synchronization technology based on WSN [3].
According to the data transmission process, it can be found that the main factors affecting the time synchronization uncertainty are [4]: Send Time, Access Time, Transmission Time, and Propagation Time. Receiving time and receiving time.

\section{TIME SyNCHRONIZATION MODEL EASE OF USE}

Each monitoring node in the wireless sensor network has its own local clock, represented by the interrupt count in its own crystal. The data shows that a typical quartz oscillator node with a frequency offset of approximately $40 \times 10-6$ (S/min) can produce a time deviation of $0.144 \mathrm{~s}$ within $1 \mathrm{~h} \mathrm{[5].} \mathrm{The} \mathrm{local}$ clock model of any monitoring node $i$ at time $t$ can be expressed as:

$$
\mathrm{T}_{i}(t)=k \int_{t 0}^{t} f_{i}(t) d t+t_{i}\left(t_{0}\right)
$$

Where fi(t) is the actual frequency of the local clock of node $\mathrm{i}$ and there is a deviation from $\mathrm{f} 0 ; \mathrm{k}$ is a constant, ie, the inverse of frequency $1 / \mathrm{f} 0$; t 0 is the timing initial time; ti(t0) is the node can be regarded as the value of $\mathrm{fi}(\mathrm{t} 0)$ in a short time at t0. Therefore, the clock information of the node can be expressed as:

$$
\mathrm{T}_{i}(t)=k_{i}\left(t-t_{0}\right)+b_{i}
$$

Among them, $k_{i}=\frac{f_{i}}{f_{0}}$ is the relative frequency of node $\mathrm{i} ; b_{i}=t_{i}\left(t_{0}\right)+b_{0}$ is the local time of the node at time t0, also known as the initial phase. Therefore, the local clock information between any two nodes $i$ and $j$ can be expressed as:

$$
t_{j}(t)=a_{i j} t_{i}(t)+b_{i j}+\varepsilon
$$

Among them, ${ }^{a_{i j}}=\frac{f_{i}}{f_{j}}$ Is the relative frequency offset between nodes; ${ }_{i j}=b_{j}-a_{i j} b_{j}$ is the relative clock offset between nodes; the time difference for the receiving node to receive the same beacon. According to formula 3, at that time, the clocks of node $\mathrm{i}$ and node $\mathrm{j}$ are exactly the same. 
According to the above time model, the task of time synchronization is to estimate the frequency offset and the phase offset value so as to adjust the clock of the receiving node. The frequency of the clock drifts with time, and the corrected node clock will reappear after a certain period of time Therefore, the synchronization algorithm requires periodically sending synchronization messages to compensate for frequency offset and phase offset in order to maintain the accuracy of synchronization. The estimated initial state aij and bij values can be estimated using the observation data tim, tjm, which is:

$$
\left\{\begin{array}{c}
n \bar{t}_{i m}=n \hat{b}_{i j}+n \hat{a}_{i j} \bar{t}_{i m} \\
\sum_{m=1}^{n} t_{i m} t_{j m}=n \bar{t}_{i m} \hat{b}_{i j}+\sum_{m=1}^{n} t_{i m}{ }^{2} \hat{a}_{i j}
\end{array}\right.
$$

among them,

$$
\bar{t}_{j m}=\frac{1}{n} \sum t_{j m} \bar{t}_{i m}=\frac{1}{n} \sum t_{j m}
$$

therefore, The estimated value of $\Delta$ is:

$$
\begin{gathered}
\hat{a}_{i j}=\frac{\sum\left(t_{j m}-\bar{t}_{j}\right)\left(t_{i m}-\bar{t}_{i}\right)}{\sum\left(t_{j m}-\bar{t}_{j}\right)^{2}} \\
\hat{b}=\bar{t}_{i}-\hat{a}_{i j} \bar{t}_{j}
\end{gathered}
$$

The clock can be adjusted based on the estimated value to complete the synchronization between the receiving nodes [6].

\section{TIME SYNCHRONIZATION MECHANISM}

After years of research by the researchers, three basic WSN time synchronization techniques are proposed, including: Receiver-Receiver Synchronization (RR mechanism) reference broadcast time synchronization mechanism, sender-receiver (Sender-Receiver) Synchronization, SR mechanism) Information exchange time synchronization mechanism, Receiver-only Synchronization (SO mechanism) time synchronization mechanism [7].

\section{A. RR Mechanism}

The receiver-recipient mechanism is implemented by compensating for the time difference between arriving at different nodes after the message is sent.

The sending node sends the broadcast information. The two receiving nodes in the broadcast domain use their own local clock to record the arrival time of the information, and exchange information to obtain the offset to achieve synchronization. This method eliminates the error caused by the sending node, and only adjusts the offset between the receiving nodes, which improves the accuracy of synchronization to some extent. However, the exchange of information between each child node brings a large amount of data processing, which may cause delays in the transmission of information and increase the communication load.

The reference broadcast clock protocol (RBS) follows the RR mechanism. The RBS algorithm adopts the method of broadcasting beacons to achieve the synchronization of the receiving nodes to eliminate the uncertainty of the sending nodes. Multiple broadcasts can improve the accuracy of synchronization and have a good implementation effect. Of course, this synchronization algorithm needs a broadcast channel and cannot be used for point-to-point communication. In addition, the amount of data exchange is huge, the synchronization time is long, and the energy consumption is large.

\section{B. SR Mechanism}

The sender-receiver mechanism joins the information of the sending node. This algorithm has a low utilization rate and requires a large amount of information to be sent, received, and exchanged, requiring a large amount of traffic and storage space.

\section{1) Unidirectional information exchange:}

The idea of the sender-recipient one-way information exchange mechanism is that the reference node, like the child node to be synchronized, sends a local clock containing its own timestamp. The child node to be synchronized accepts and records the arrival time, and then according to its own clock information. Calculate the delivery delay of the message and adjust it to achieve synchronization. The typical algorithm under this mechanism is the FTSP (Flooding Time Synchronization Protocol) algorithm.

The FTSP algorithm uses the Hongfan broadcast beacon to establish time synchronization across the entire network, consumes a lot of power, and is easily affected by the selection of reference nodes. Reducing the uncertainty of time delay requires a certain amount of algorithm analysis and support. In practice, it is widely used due to its low complexity, high precision, and low power consumption [5].

\section{2) Two-way information exchange:}

The idea of a sender-receiver bidirectional information exchange mechanism is that the child node to be synchronized sends a synchronization request containing the local clock information to the child node to be synchronized, the reference node records the arrival time of the information and replies to the confirmation, and then the child node to be synchronized is based on The acknowledgment time is calculated by calculating the offset between the local clock information and the clock of the reference root node, and adjustments are made to achieve synchronization. The typical algorithm under this mechanism is the Time Synchronization Protocol for Sensor Network (TPSN) algorithm.

The Sensor Time Synchronization Protocol (TPSN) implements time synchronization among all nodes in the WSN across the entire network. The TPSN algorithm reduces the 
synchronization measurement error of the access time on the one hand, and adds the beacon when the message is sent to the wireless channel; on the other hand, the calculation accuracy is improved, the average delay is calculated by using the bidirectional transmission, and the propagation time and the reception time are taken into consideration. Different, with a certain degree of scalability. However, it does not consider the failure problem of the reference node. When the new node joins, it needs to initialize the level discovery phase. Its static characteristics reduce the robustness of the algorithm and the energy cannot be used effectively.

\section{RO Mechanism}

The time synchronization mechanism of receiving only focuses on improving the accuracy of instantaneous synchronization. The principle is that the sender sends a message containing its time information to the receiver. The sender and the receiver use the MAC layer to mark the timestamp, and the receiver sends The time information structure of the terminal is matched with the time of the local clock information, so as to adjust the local clock value, the DMTS (Delay Measurement Time Synchronization) algorithm. This algorithm is simple to implement, consumes less power and eliminates other uncertainties outside the propagation delay time, but the synchronization accuracy is low.

\section{D.Hybrid synchronization mechanism}

It is difficult for these three synchronization mechanisms to take into account the scalability, energy consumption, and synchronization accuracy at the same time. In a multi-hop wireless sensor network, synchronization errors tend to accumulate with jump distances and cannot be applied. For its improvement, on the one hand, it can be improved from the algorithm, and the distributed WSN time synchronization mechanism is used to introduce time offset and phase offset between pairs of sensor nodes, such as maximum likelihood estimation, linear minimum variance estimation, Bayes estimation, Kalman filtering, etc.; On the other hand, it can be improved from the whole network time synchronization protocol, adopting a centralized WSN time synchronization mechanism to achieve the purpose of improving the synchronization accuracy, such as the HRTS (Hierarchy Referencing Time Synchronization) algorithm is Based on the TPSN model and the RBS model, an algorithm that combines the advantages of the two, a three-information transmission within a broadcast domain can realize the synchronization of all nodes, synthesize the advantages of these two methods, reduce energy consumption, and improve synchronization. Accuracy.

In the hierarchical reference time synchronization protocol (HRTS), when one of the child nodes sends a time request, all related nodes are requested and the value of the received local clock is recorded.

The HRTS algorithm uses a hierarchical broadcast to achieve synchronization, but the tiering is more reasonable, and there are groupings between child nodes, which improves accuracy, speed, and storage space while also increasing sensitivity.

\section{COMPARISON AND ANALYSIS OF TIME SYNCHRONIZATION ALGORITHMS}

Summarize the characteristics of the above several algorithms are as follows: RBS algorithms, Eliminate sender's time uncertainty, clock adjustment does not affect the time offset calculation, Large energy consumption, Algorithm complex, applicable to wireless sensor networks and wired networks change; FTSP algorithms appropriate topological mechanism and redundancy processing, low power consumption, high precision,need corresponding algorithm support, applicable to military affairs; TPSN algorithm, eliminate sender's timeuncertainty, accuracy of several hundred microseconds, supports external clock source, large energy consumption, not adapting to dynamic changes, high complexity, Stable network with high synchronization accuracy; DMTS algorithms, Simple implementation, low power consumptio, low precision, WSN network with low complexity and low accuracy requirement; HRTSalgorithms, low power consumption, high precision, reduced traffic, high energy consumption wireless sensor network, with high environmental requirements.

In the Matlab environment, the measurement errors of the above synchronization algorithms are simulated and analyzed. The wireless communication distance is set to $30 \mathrm{~m}$ in a $50 \mathrm{~m} \mathrm{x}$ $50 \mathrm{~m}$ square area and the synchronization period is 50 . The phase deviation between the monitoring nodes is $[-50,50] \mu \mathrm{s}$, and the frequency deviation satisfies the normal distribution of $\mu=1, \sigma 2=0.001$. The result is shown in Figure I.

The comparison analysis shows that the synchronous measurement errors of RBS, DMTS, FTSP, TSPN, and HRTS algorithms are successively reduced. The maximum measurement errors are $37.55 \mu \mathrm{s}, 34.65 \mu \mathrm{s}, 29.79 \mu \mathrm{s}, 26.27 \mu \mathrm{s}$, and $18.77 \mu$ s respectively. The HRTS algorithm is the most realized. it is good.

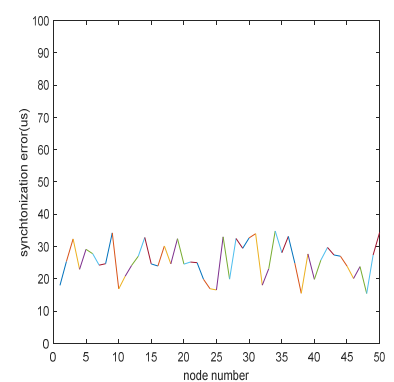

(1)RBSALGORITHM

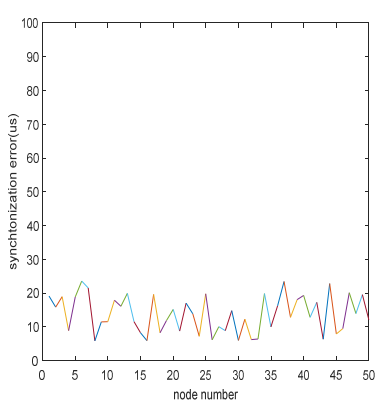

(3)TPSNALGORITHM

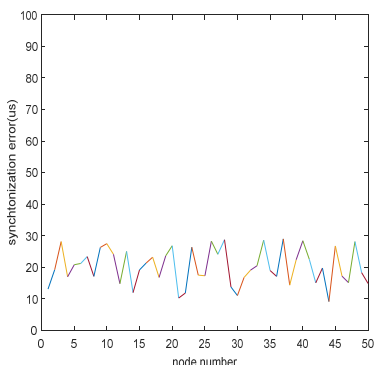

(2)FTSPALGORITHM

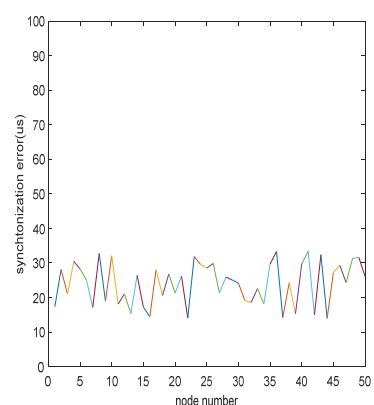

(4)DMTSALGORITHM 


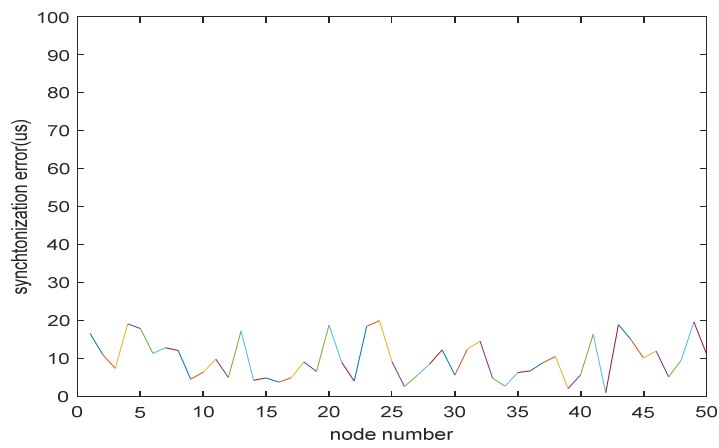

FIGURE I. TIME SYNCHRONIZATION MEASUREMENT ERROR OF FIVE ALGORITHMS

\section{CONCLUSION}

This article mainly introduces the time synchronization technology in WSN. Firstly, it analyzes the application challenges of synchronization technology at the present stage. Then it gives the clock model of nodes in WSN, and introduces the basic types of time synchronization technology respectively: RR mechanism, SR Mechanism, RO mechanism, hybrid synchronization mechanism, analysis of the synchronization mechanism principle, scope of application, and advantages and disadvantages of different time synchronization algorithms ineach mode, and the error analysis of these mechanisms was simulated by MATLAB, and the HRTS algorithm was compared afterwards The time error of the maximum deviation is the smallest and the degree of implementation is best. Finally, the HRTS algorithm is selected as the synchronization mode of the WSN network.

\section{REFERENCES}

[1] Shen Jiajun. Vulnerability Analysis of IEEE1588 Precision Clock Synchronization Protocol [D]. Zhejiang: Zhejiang University, 2015.

[2] Shi Zhilu. Research on network coding compression and decoding algorithms in wireless sensor networks [D]. Jiangsu: Jiangsu University of Science and Technology, 2015.

[3] Li Qian. Research and implementation of clock synchronization technology in wireless sensor networks [D]. Nanjing: Nanjing University of Posts and Telecommunications, 2014.

[4] Dun Wentao, Bi Qingsheng, Hui Xianghui, Xia Bin, Cai Wei, Yang Zhaoyan, Wu Haiyan. Application of Wireless Sensor Network in Digital Agriculture[J]. Science and Technology Vision, 2012,(29):123$124+114$.

[5] Cao Xiaojia. Research on time synchronization of wireless sensor networks for mechanical vibration monitoring [D]. Chongqing University, 2013.

[6] Yang Wenhuan, Sun Jiuyun, Wang Kuanyin. Design of remote monitoring system for coal mine environment based on ZigBee and GPRS [J]. Metal Mine, 2015, (06): 125-129.

[7] Guo Xiaoqin, Sun Yigang, Chen Weixing. Study on Key Technologies of Wireless Sensor Network Monitoring System for Airport Scene[J] Computer Engineering and Design, 2009,(22):5106-5108+5143.

[8] Mo Yong. Research on wireless sensor network time synchronization based on Kalman consistent filter [D]. Guilin University of Electronic Technology, 2013 